\title{
Peso e condição corporal, contagem de OPG e perfil metabólico sanguíneo de ovelhas da raça Santa Inês no periparto, criadas na região da Baixada Litorânea do Estado do Rio de Janeiro
}

\section{Body weight and body condition, EPG couting and blood metabolic profile of Santa Ines ewes in the peripartum kept in the coastal plains of Rio de Janeiro}

\author{
Elyzabeth da Cruz Cardoso, ${ }^{*}$ Daniel Rocha Oliveira, ${ }^{* *}$ Antonio Prieto Dourado, ${ }^{* *}$ Cláudio Vieira Araújo, ${ }^{* \star *}$ \\ Enrico Lippi Ortalani, ${ }^{* * *}$ Felipe Zandonadi Brandão*
}

\begin{abstract}
Resumo
O presente trabalho descreveu mudanças do peso, da condição corporal (CC), da contagem de ovos por grama (OPG) de fezes e do perfil metabólico de ovelhas da raça Santa Inês em diferentes tempos do periparto. Análise de variância foi efetuada considerando $5 \%$ de significância e aplicação da equação de regressão nas variáveis significativas. A condição corporal diminuiu após o parto. As concetrações de hemoglobina diminuíram entre T+20 e T+30 coincidente com o aumento do OPG. As concentrações de albumina e ureia apresentaram equações de regressão lineares com decréscimo dos valores do pré-parto para o pós-parto. As equações de regressão para o beta-hidroxibutirato, cálcio, fósforo e magnésio apresentaram um comportamento quadrático. As concentrações de beta-hidroxibutirato e cálcio foram mais elevados tanto nos dias que antecederam quanto nos que sucederam ao parto. Os valores mais elevados de fósforo foram observados nos tempos próximos ao parto, decrescendo aos 30 dias do pós-parto. O magnésio apresentou um aumento de seus valores no pósparto. A manutenção do ECC entre 3,0 e 2,5 no período periparto garantiu parâmetros hematológicos compatíveis com a produção e saúde dos animais. Os valores baixos de albumina sérica e ureia evidenciaram um balanço proteico inadequado. O OPG foi sensível a variações no periparto.
\end{abstract}

Palavras-chave: ovelhas, sangue, perfil metabólico, gestação, lactação.

\begin{abstract}
Changes in weight, body condition (BC) and eggs per gram (EPG) in the feces and the metabolic profile of Santa Ines ewes in different peripartum times were described in this study. Analysis of variance was used considering a $5 \%$ significance and the application of regression equation for the significant variables. Body condition declined after birth. Hemoglobin concentrations decreased between $T+20$ and $T+30$, coincidently with the increase of EPG. Albumin and urea concentration had linear regression equations together with the decline of pre- and postpartum values. Regression equations for betahydroxybutyrate, calcium, phosphorus and magnesium had quadratic behavior. Beta-hydroxybutyrate and calcium concentrations were higher in the days pre- and postpartum. Highest values of phosphorus were observed in the times close to birth, declining at 30 days postpartum. Increased values of magnesium were observed postpartum. Maintenance in ECC between 3.0 and 2.5 in the peripartum maintained the blood parameters compatible with the production and health of these animals. Low values of serum albumin and urea evidenced inadequate protein balance. EPG was sensitive to peripartum variations.
\end{abstract}

Keywords: ewes, blood, metabolic profile, pregnancy, lactation.

\section{Introdução}

No Brasil, apesar da carne ovina ainda ocupar uma parcela muito pequena no ranking do consumo de carnes, na última década, a ovinocultura vem ganhando expressividade, tendo como principal produto o cordeiro (Granados et al., 2006). De acordo com os dados descritos no Jornal Cabra \& Ovelha (2008), somente no $1^{\text {o }}$ semestre de 2007 foram importadas do Uruguai, Chile e Argentina cerca de 2.900 toneladas de carne ovina ao custo de US\$ 6 milhões.

\footnotetext{
*Professores da Faculdade de Veterinária da Universidade Federal Fluminese. Rua Vital Brazil Filho, 64. Niterói. RJ. 24230-340. E-mail para contato: elyzabethcardoso@hotmail.com

**Alunos do Programa de Pós-Graduação em Medicina Veterinária (Clinica e Reprodução Animal) - FV/UFF

***Professor do Instituto de Saúde e Produção Animal da UFRA.

**** Professor da Faculdade de Veterinária da USP
} 
Um dos problemas que restringem a ovinocultura brasileira é o fato de que as principais raças estrangeiras destinadas ao abate têm características reprodutivas pouco favoráveis, demonstrando-se poliéstricas estacionais. Contudo, raças nacionais, entre elas a Santa Inês, vêm apresentando potencial positivo para a produção nas regiões tropicais e subtropicais, visto que as fêmeas são poliéstricas não estacionais, e mesmo não sendo sua produtividade igual à das raças especializadas (Mexia et al., 2004). Animais da raça Santa Inês são de grande porte, produzem boa carcaça, contêm pele forte e resistente e são adaptadas às diferentes condições climáticas. As ovelhas se destacam pela habilidade materna e pela capacidade leiteira, observandose frequentemente partos gemelares no rebanho.

O período de gestação das ovelhas é sempre bastante crítico, devendo-se dar atenção às questões nutricionais. Isso porque a condição de gestação eleva as necessidades alimentares, especialmente durante as últimas seis semanas, quando se acelera o desenvolvimento fetal e completam-se aproximadamente $70 \%$ do seu crescimento (Russel, 1979). Nessa fase também ocorre incremento das necessidades maternas de nutrientes para o desenvolvimento do úbere e da própria manutenção (El-Sherif e Assad, 2001). O tipo de gestação, a nutrição e as estações do ano podem ser responsáveis por alterações metabólicas em fêmeas em atividade reprodutiva, por isso é de extrema importância utilizar mecanismos que possam monitorar o processo metabólico nas diferentes fases fisiológicas (Brito et al., 2006; Balikci et al., 2007).

Desequilíbrios metabólicos são definidos por Payne y Payne (1970) como doenças de produção e podem surgir quando ocorre desequilíbrio entre os ingressos e egressos dos nutrientes. No sangue, os perfis bioquímicos têm sido utilizados para obtenção de informações sobre a condição nutricional dos animais e na avaliação dos fenômenos reprodutivos, sobretudo no periparto, definido como o período de transição entre as três semanas anteriores e posteriores ao parto (Ingraham e Kappel, 1988). Dietas com restrição proteico-energética na fase inicial da lactação levam à diminuição da produção láctea e do escore corporal, bem como o aumento do intervalo parto/concepção, enfermidades do puerpério e, consequentemente, maior intervalo entre partos (Rossato et al., 1999).

Del Valle et al. (1983), ao estudarem as variações da composição sanguínea de ovelhas no pré e no pós-parto, concluíram que os componentes que melhor expressaram as variações do estado nutricional foram a hemoglobina (HB), o hematócrito (HTC) e a glicemia, os quais diminuíram quando os requerimentos nutricionais aparentemente não foram preenchidos. Além disso, as variações de HB e HC apresentaram um alto grau de correlação significativa com as variações de peso vivo e da condição corporal.

O monitoramento dos padrões proteico, energético e mineral em ovelhas é uma ferramenta de grande importância para a adequação alimentar e da avaliação da condição metabólica, considerando, sobretudo a pressão do processo de intensificação da produtividade (González, 2000; Ribeiro, 2004; Caldeira, 2005).

Como existem poucas informações a respeito do perfil metabólico em ovelhas da raça Santa Inês, e por ser a ovinocultura brasileira uma atividade comumente secundária à bovinocultura, o presente trabalho objetivou reportar as mudanças do peso, do escore corporal e do perfil metabólico sanguíneo na raça, em fêmeas no periparto em uma propriedade da região da Baixada Litorânea do Estado do Rio de Janeiro.

\section{Material e métodos}

O trabalho foi realizado em uma propriedade particular do município de Cachoeiras de Macacu, utilizando-se nove ovelhas da raça Santa Inês, adultas, clinicamente sadias, criadas em regime semi-intensivo em pastagem do gênero Brachiaria (B. brizantha e B. humidicola). Trinta dias antes da parição, as ovelhas permaneceram confinadas no aprisco recebendo capim picado (Penisetum purpureum) acrescido de concentrado comercial duas vezes ao dia. Água e mistura mineral foram fornecidas à vontade nas duas condições de manejo a que os animais foram submetidos. As ovelhas foram vacinadas conforme calendário sanitário e a desverminação realizada 30 dias antes do parto, no parto e 30 dias após o parto $(5 \mathrm{ml} / 30 \mathrm{~kg}$ de peso vivo de Aldazol com cobalto - Valléeß).

O estudo abrangeu o período compreendido entre 30 dias antes $(T-30)$ e 30 dias após o parto $(T+30)$, onde T0 correspondeu ao parto. Nos intervalos predeterminados (tabela 1), pela manhã, com os animais em jejum prévio de 12 horas, foram colhidas as amostras de sangue, realizadas as pesagens e avaliações do escore de condição corporal (ECC) individual seguindo as descrições de Suiter (2006). Também foram colhidas fezes de cada um dos animais no início, meio e no final do período estudado, as quais foram acondicionadas para análises parasitológicas.

O ganho de peso (GP) foi calculado a partir das diferenças entre os pesos iniciais e finais dos animais dentro de cada intervalo de pesagem realizada.

O sangue foi colhido por meio de venopunção jugular e distribuído em dois tubos a vácuo, um contendo EDTA a 10\% para obtenção de plasma e o outro sem anticoagulante para obtenção do soro.

Imediatamente após a colheita de sangue foram efetuadas as análises da hemoglobina (HG) e de hematócrito (HTC) através das técnicas de espectrofotometria e de microhematócrito descritas por Jain (1993).

Tabela 1: Períodos considerados para a determinação do perfil metabólico de ovelhas da raça Santa Inês no periparto

\begin{tabular}{ll}
\hline \multicolumn{1}{c}{ Períodos } & \multicolumn{1}{c}{ Intervalo } \\
\hline T-30 & 30 a 21 dias antes do parto \\
T-20 & 20 a 11 dias antes do parto \\
T-10 & 10 a 6 dias antes do parto \\
T- 5 & 5 a 1 dias antes do parto \\
T0 & Dia do parto a 4 dias após o parto \\
$T+5$ & 5 a 9 dias do pos parto \\
$T+10$ & 10 a 19 dias do pós parto \\
$T+20$ & 20 a 29 dias do pós parto \\
$T+30$ & Acima de 30 dias do pós parto \\
\hline
\end{tabular}


As análises plasmáticas incluíram a determinação da glicose (GLI), ureia (URE) e beta-hidroxibutirato (BHB). As sorológicas, o cálcio $(\mathrm{Ca})$, o fósforo $(\mathrm{P})$, o magnésio $(\mathrm{Mg})$, a proteína total (PT), a albumina (ALB) e a globulina (GLOB). Com exceção do BHB, as demais análises foram realizadas no Laboratório de Patologia Clínica Veterinária do Hospital Veterinário da Universidade Federal Fluminense, utilizando-se o espectrofotômetro colorimétrico (BIOPLUS Bio-200) e kits comerciais. Os valores da globulina sérica foram obtidos através da subtração dos valores obtidos de proteína total e albumina séricas. A dosagem de BHB foi realizada no Laboratório de Doenças Metabólicas da Escola de Veterinária da Universidade de São Paulo pelo método enzimático cinético UV utilizando o Analisador Bioquímico Automatizado Liasys.

As amostras individualizadas das fezes foram colhidas diretamente da ampola retal e refrigeradas a $5^{\circ} \mathrm{C}$ para transporte ao laboratório, onde foram imediatamente efetuadas a determinação da contagem de ovos de helmintos por grama de fezes (OPG).

O período de colheita das amostras ocorreu entre os meses de janeiro a abril de 2008. Uma vez realizadas todas as análises, os resultados foram agrupados em função do dia do parto, para então definir os períodos do periparto (Tabela 1). As médias das diferentes respostadas estudadas foram comparados pelo SAS através de análise de variância com formulação da equação de regressão para as variáveis significativas $(p<0,05)$.

\section{Resultados e discussão}

Os valores médios e desvios-padrões das variáveis estuda-das nos tempos referentes ao período periparto (T-30, T-20, T-10, T-5, T0, T+5, T+10, T+20, T+30) são apresentados na Tabela 2. Observou-se significância estatística $(P<0,05)$ para as variáveis ECC, HG, ALB, $\mathrm{BHB}, \mathrm{URE}, \mathrm{Ca}, \mathrm{P}$ e $\mathrm{Mg}$. As equações de regressão destas variáveis encontram-se descritas nas Figuras de 1 a 8 , respectivamente. ALB sérica e URE plasmática apresentaram equações lineares, enquanto o ECC, HG e BHB plasmático e $\mathrm{Ca}, \mathrm{P}$ e $\mathrm{Mg}$ séricos apresentaram equações quadráticas.

O ECC médio das ovelhas decresceu após o parto $(p<0,05)$ conforme houve evolução da lactação (Figura 1), chegando a valores abaixo de 3,0. Ribeiro (2004) verificou declínio da condição corporal de ovelhas gestantes, chegando a valores críticos por volta do parto e no período de amamentação. Considerando que o feto se desenvolve acentuadamente durante o último terço da gestação, havendo mobilização de reservas corporais maternas para compensar o déficit nutricional, recomenda-se que a dieta deva ser compensada para que se adapte a esse estágio fisiológico mais sensível.

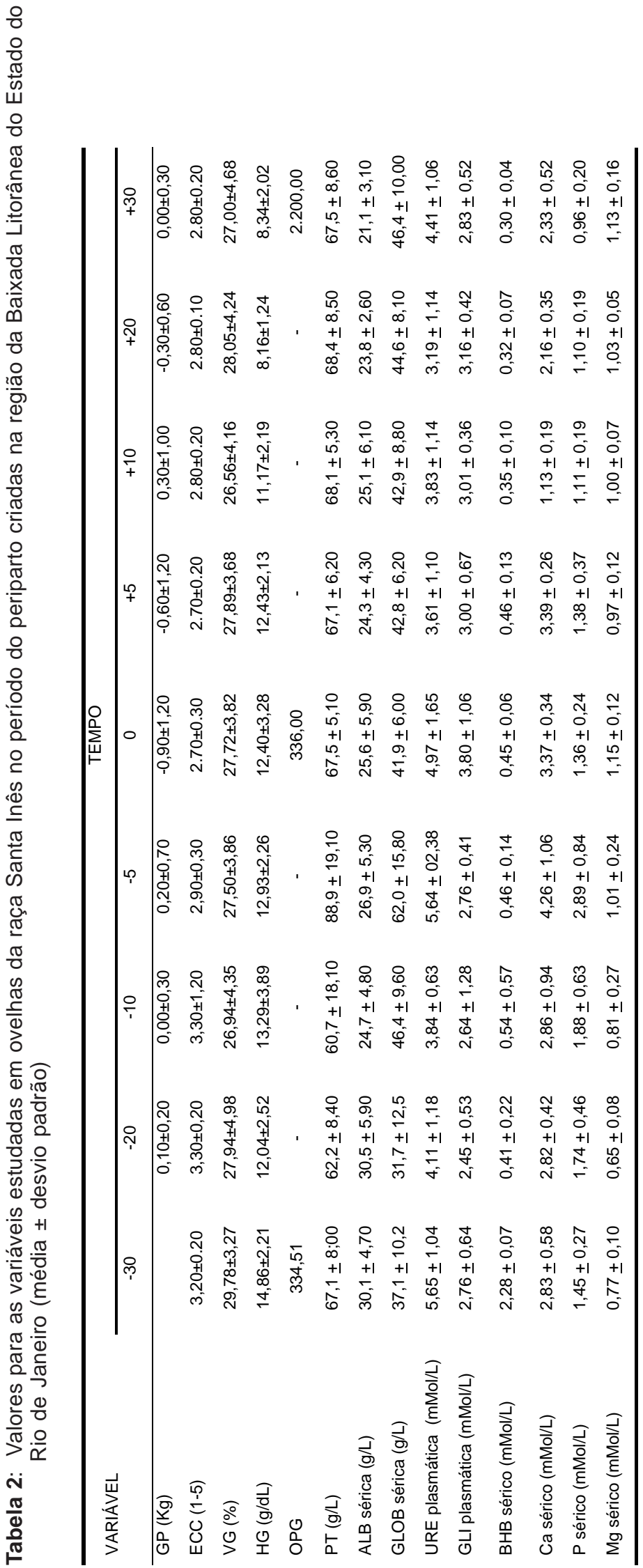




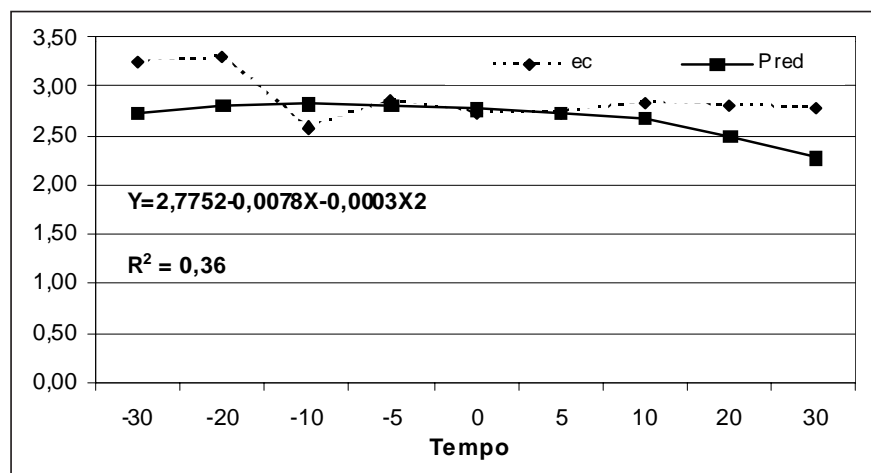

Figura 1: Gráfico de regressão para escore de condição corporal de ovelhas da raça Santa Inês no período periparto criadas na região da Baixada Litorânea do Estado do Rio de Janeiro.

O perfil hematológico é um dos parâmetros que se altera com maior facilidade e velocidade no período periparto. Brito et al. (2006) também relataram diminuição do hematócrito conforme o avanço da gestação, justificada pela hemodiluição fisiológica da gestação. No presente estudo, podemos supor que a redução mais acentuada da curva de HG nos tempos $\mathrm{T}+20$ e T+30 (Figura 2) possa estar relacionada com a elevação do OPG no pós-parto. Sasa et al. (2008), ao avaliarem o grau de infecção helmíntica (detectada em OPG) em ovelhas da raça Santa Inês, verificaram diferenças significativas entre os períodos. Do mesmo modo, conforme observado no presente estudo o período do pós-parto apresenta-se susceptível a infestações endoparasitárias.

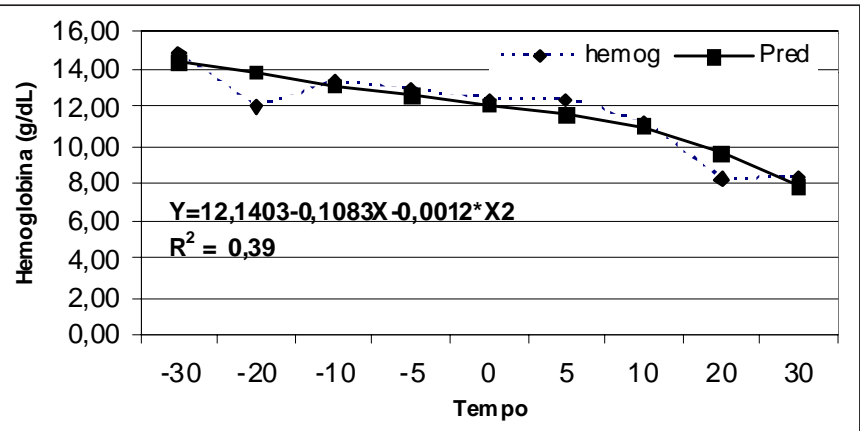

Figura 2: Gráfico de regressão para hemoglobina sanguínea de ovelhas da raça Santa Inês no período periparto criadas na região da Baixada Litorânea do Estado do Rio de Janeiro.

Os valores da ALB sérica decresceram de acordo com o tempo estudado (Figura 3). Os valores médios a partir do tempo T-10 ficaram abaixo da margem inferior dos valores de referência, 26 a 42g/L, descritos por Contreras et al.(2000). Segundo Caldeira (2005), a ALB é um indicador de longos períodos de restrição proteica e com isso é um fator muito relacionado com o processo alimentar. Assim, pode-se atribuir o declínio das quantidades deste elemento à má nutrição proteica neste período.

Os valores plasmáticos de URE foram próximos ao limite inferior da referência, 4,0 a 10,0 mMol/L (CONTRERAS et al., 2000), apresentando um comportamento decrescente ao lon-

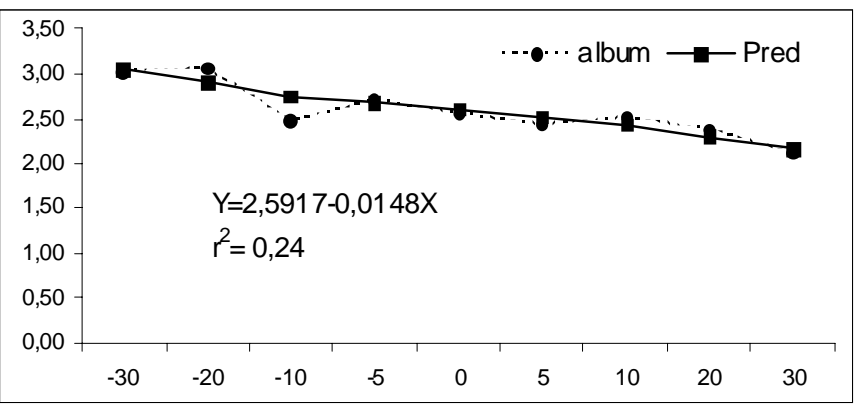

Figura 3: Gráfico de regressão da albumina sérica de ovelhas da raça Santa Inês no período periparto criadas na região da Baixada Litorânea do Estado do Rio de Janeiro.

go do período do periparto (Figura 4). Estes resultados estão concordantes com os encontrados por Ribeiro (2004), assim como as alterações observadas na ALB, a diminuição da URE reflete a real deficiência de proteína na alimentação frente às necessidades impostas pelo estado fisiológico dos animais.

Os resultados de GLI plasmática encontrados foram coerentes com os valores de referência de 2,4 a $4,4 \mathrm{mMol} / \mathrm{L}$, descritos por Contreras et al. (2000). Ficou claro que os processos de manutenção da glicemia e/ou o aporte de carboidratos na alimentação foi adequado em todos os momentos.

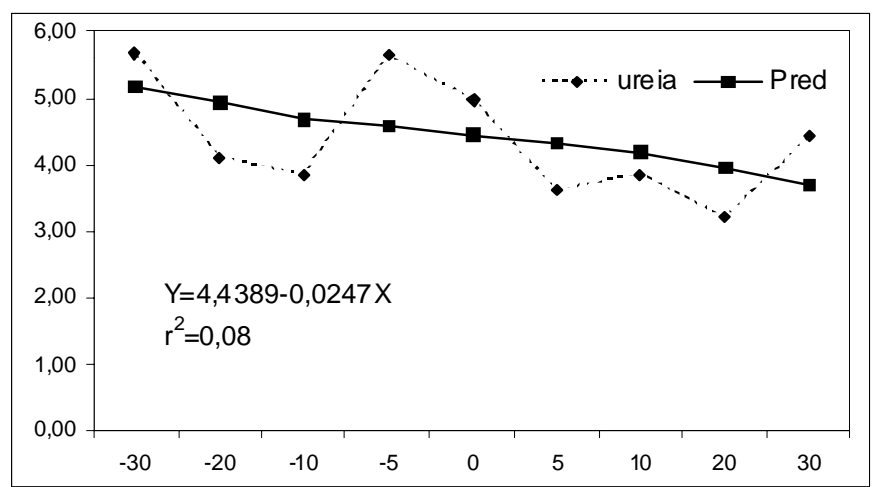

Figura 4: Gráfico de regressão da ureia plasmática de ovelhas da raça Santa Inês no período periparto criadas na região da Baixada Litorânea do Estado do Rio de Janeiro.

Os valores de BHB plasmáticos, apesar de encontrarem-se dentro dos limites de referência, $0,6 \mathrm{mmol} / \mathrm{L}$ (CONTRERAS et al., 2000), apresentaram valores mais elevados no intervalo que compreendeu os dias que antecederam e sucederam imediatamente o parto (Figura 5). Esse comportamento gráfico refletiu a provável mobilização de outros elementos que não os carboidratos para atender as necessidades de energia das ovelhas no parto. Em associação, devem-se incluir os fatores fisiológicos de diminuição da ingestão de matéria seca e desenvolvimento do úbere (El-Sherif e Assad, 2001) que normalmente ocorrem nessa fase, diminuindo a quantidade de energia disponível.

Segundo González (2000), o Ca está intimamente ligado ao metabolismo do periparto, porém não é um bom indicador do estado nutricional pelo forte controle endócrino isocalcê- 
mico. Os valores de $\mathrm{Ca}$ sérico encontrados no pré-parto e início da lactação demonstram médias acima dos valores de referência descritos por Contreras et al. (2000), 2,1 a 2,5mmol/L, em especial nos tempos T-5, T0 e T+5 (Figura 6). Possivelmente o aumento dos valores séricos tenha ocorrido pela mobilização de $\mathrm{Ca}$ para os processos do parto e da lactação.

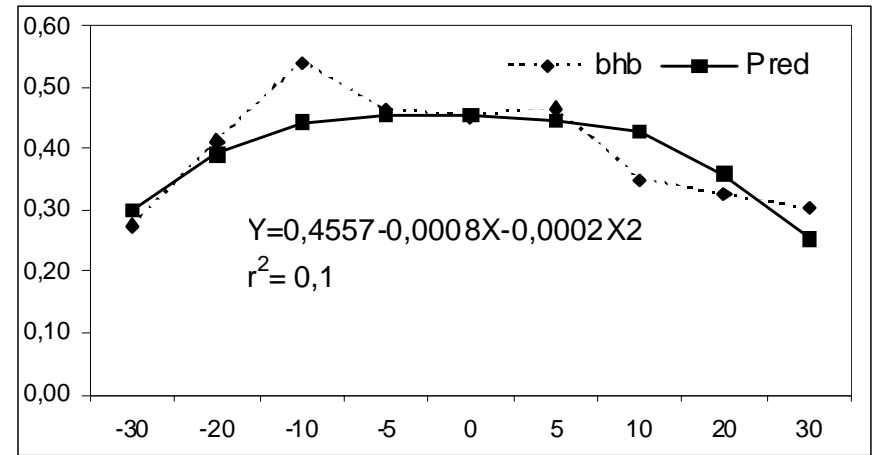

Figura 5: Gráfico de regressão do beta-hidroxibutirato plasmático de ovelhas da raça Santa Inês no período periparto criadas na região da Baixada Litorânea do Estado do Rio de Janeiro

Ainda de acordo com González (2000), a relação Ca:P no leite de vacas é de quase 1:1. No entanto, a relação desses elementos nos alimentos é de 2:1, o que levaria a diminuição das quantidades circulantes. Neste estudo verificaram-se médias dentro dos valores de referência, e um comportamento decrescente do gráfico de correlação (Figura 7), fato que está de acordo com as afirmações de Ribeiro (2004), que encontrou valores decrescentes de $\mathrm{P}$ sanguíneo de acordo com a evolução da lactação.

Os valores de $\mathrm{Mg}$ sérico mantiveram-se dentro do intervalo de referência $(0,7-1,1 \mathrm{mmol} / \mathrm{l})$ sugerido por Contreras et al. (2000). A Figura 8 mostra que os valores de Mg foram mais elevados no final do período de periparto. Segundo González (2000) e Ribeiro (2004), os valores deste mineral são influenciadas pelo balanço entre sua ingestão e a sua eliminação, com isso foi possível concluir que o balanço desse elemento ocorreu adequadamente, muito embora sua mobilização possa estar ocorrendo para atender as necessidades fisiológicas da lactação.

\section{Conclusões}

Nas condições do presente estudo, podemos concluir:

- a concentração de hemoglobina foi o parâmetro mais sensível ao balanço metabólico negativo observado no pico de lactação;

- ovelhas da raça Santa Inês em condição corporal entre 3,0 e 2,5 no período do periparto são capazes de garantir parâmetros hematológicos (volume globular e hemoglobina) compatíveis com a produção e saúde;

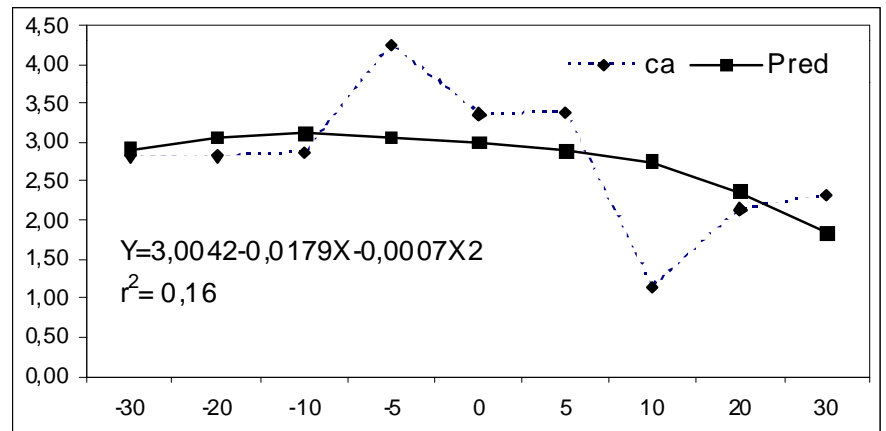

Figura 6: Gráfico de regressão do cálcio sérico de ovelhas da raça Santa Inês no período periparto criadas na região da Baixada Litorânea do Estado do Rio de Janeiro.

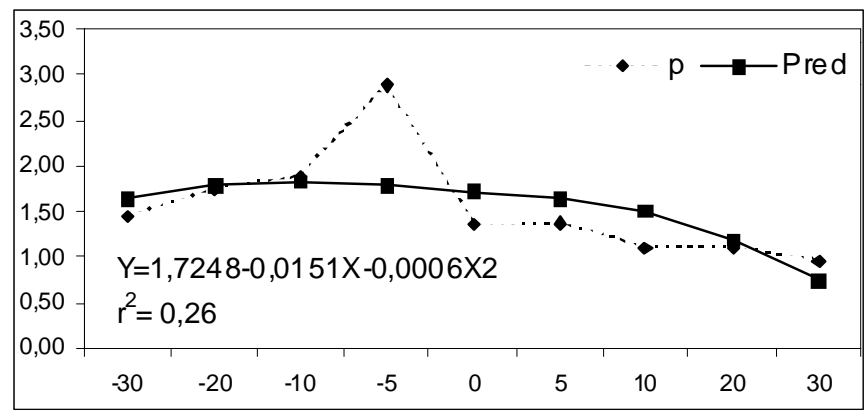

Figura 7: Gráfico de regressão do fósforo sérico de ovelhas da raça Santa Inês no período periparto criadas na região da Baixada Litorânea do Estado do Rio de Janeiro.

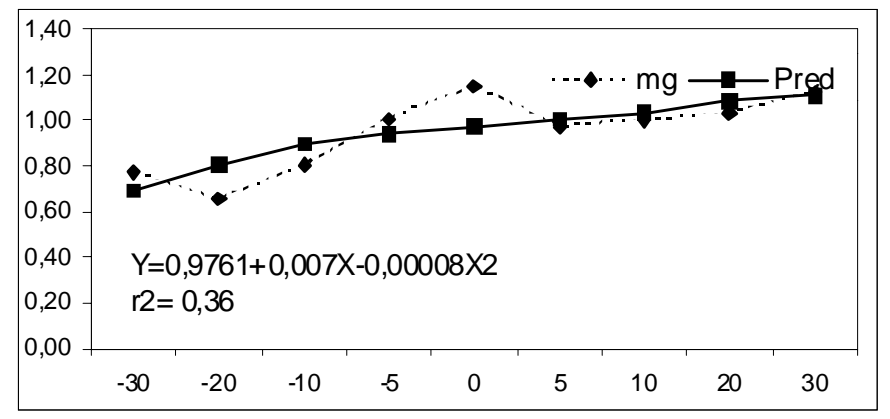

Figura 8: Gráfico de regressão do magnésio de ovelhas da raça Santa Inês no período periparto criadas na região da Baixada Litorânea do Estado do Rio de Janeiro.

- valores baixos de albumina sérica e ureia plasmática evidenciaram um balanço proteico inadequado, não contemplando as necessidades nutricionais dos animais no periparto, e

- a contagem de ovos de helmintos por grama de fezes foi sensível a variações no periparto, devendo-se atentar para os cuidados de profilaxia e terapia contra endoparasitas que ocorrem no período. 


\section{Referências}

BALIKCI, E.; YILDIZ, A.; GÜRDOGAN, F. Blood metabolite concentration during pregnancy and postpartum in Akkaraman ewes. Small Ruminant Research. v. 67, p. 247-251, 2007.

BRITO, M.A.; GONZÁLEZ, F.D.; RIBEIRO, L.A., CAMPOS, R., BARBOSA, P.R.; BERGMAN, G. Composição do sangue e do leite em ovinos leiteiros do sul do Brasil: variações na gestação e lactação. Ciência Rural. v. 36, n. 3, p. 1-7. 2006.

CALDEIRA, R.M. Monitoração da adequação do plano alimentar e do estado nutricional em ovelhas. Revista Portuguesa de Ciências Veterinárias. v. 100 (555-556), p. 125-139, 2005.

CONTRERAS, P.; WITTWER, F.; BÖHMWALD, H. Uso dos perfis metabólicos no monitoramento nutricional de ovinos. In: GONZALEZ, F.H.D.; BARCELLOS, J.O.; OSPINA, H.; RIBEIRO, L.A.O. (Eds.) Perfil metabólico em ruminantes: seu uso em nutrição e doenças nutricionais. Porto Alegre: Gráfica da Universidade Federal do Rio Grande do Sul, 2000.

DEL VALLE, J., WITTWER, F., HERVÉ, M. Estudio de los perfiles metabólicos durante los períodos de gestación y lactancia en ovinos Romney. Archivos de Medicina Veterinaria, v. 15, p. 65-72. 1983

EL-SHERIF, M.M.A; ASSAD, F. Changes in some blood constituints of Bark ewes during pregnancy and lactation under semi arid conditions. Small Ruminant Research, v. 40, p. 269-277, 2001.

GONZÁLEZ, F.H.D. Indicadores sanguíneos do metabolismo mineral em ruminantes. In: GONZÁLEZ, F.H.D.; BARCELLOS, J.O.; OSPINA, H.; RIBEIRO, L.A.O. (Eds.) Perfil metabólico em ruminantes: seu uso em nutrição e doenças nutricionais. Porto Alegre: Gráfica da Universidade Federal do Rio Grande do Sul, 2000.

GRANADOS, L. B. C.; DIAS, A. J. B.; SALES, M. P. Aspectos gerais da reprodução de caprinos e ovinos. Universidade estadual do Norte Fluminense Darcy Ribeiro. Rio de Janeiro, 2006.
INGAHAM, R. H.; KAPEL, L. C. The tests used in profile metabolic. The Veterinary Clinics of North America, Food Animal Practice, v. 4, n. 2, p. 391-411, 1988.

.JAIN, N.C. Schalm's Veterinary Hematology. 4. ed. Philadelphia: Lea \& Febiger, 1993. $221 \mathrm{p}$.

JORNAL CABRA \& OVELHA, n. 25, p. 61, fevereiro 2008.

MEXIA, A. A.; MACEDO, F. A. F.; ALCALDE, C. R.; SAKAGUTI, E. S.; MARTINS, E. N.; ZUNDT, M.; YAMAMOTO, S. M.; MACEDO, R. M. G. Desempenho reprodutivo e produtivo de ovelhas Santa Inês suplementadas em diferentes fases da gestação. Revista Brasileira de Zootecnia, v. 33, n. 3, p. 658-667, 2004.

PAYNE, J.M.; PAYNE, S. The Metabolic Profile Test. Oxford, Oxford University Press, 1970.179 p.

RIBEIRO, L.A.O. Perfil metabólico de ovelhas Border Leicester x Texel durante a gestação e a lactação. Revista Portuguesa de Ciências Veterinárias. v. 99, n. 551, p. 155-159, 2004.

ROSSATO, W. L.; GONZÁLEZ, F.D.; DIAS, M. M.; FARIA, S. V.; RICCÓ, D. Condição metabólica e desempenho reprodutivo no pós-parto em vacas leiteiras no sul do Brasil. Revista Brasileira de Reprodução Animal, v. 23, n. 3, p. 155-156, 1999.

RUSSEL, A.J.F. The nutrition of the pregnant ewe. In: British Council. The management and diseases of sheep. Edinburg, 1979.

SAS. User's procedures guide. Version 6. 4. ed. vol. 1-2, Cary, NC:SAS Institute, Inc, 1989.

SASA, A., NEVES, E.P, CASTILHO, M.F.O., MEXIA, A.A. Infecção helmíntica em ovelhas Santa Inês no periparto criadas na região do Pantanal brasileiro. Revista Brasileira de Produção Animal. v. 49, n. 2, p. 321-326, 2008.

SUITER, J. Body condition scoring of sheep and goats. Farmnote 69/ 1994 (Reviewed July, 2006). 